\title{
Retromandibular Approach for Mandibular Sub Condylar Fracture: A Review of Literature
}

\section{Jimson Samson ${ }^{1 *}$, Harivighnesh $S^{2}$, Anandh Balasubramanian ${ }^{3}$ and Jones Jayabalan ${ }^{4}$}

${ }^{1}$ Professor and Head, Department of Oral and Maxillofacial Surgery, Tagore Dental College and Hospital, Rathinamangalam, Tamil Nadu, India

${ }^{2}$ Postgraduate Trainee, Department of Oral and Maxillofacial Surgery, Tagore Dental

College and Hospital, Rathinamangalam, Tamil Nadu, India

${ }^{3}$ Reader, Department of Oral and Maxillofacial Surgery, Tagore Dental College and

Hospital, Rathinamangalam, Tamil Nadu, India

${ }^{4}$ Senior Lecturer, Department of Oral and Maxillofacial Surgery, Tagore Dental

College and Hospital, Rathinamangalam, Tamil Nadu, India

*Corresponding Author: Jimson Samson, Professor and Head, Department of Oral and Maxillofacial Surgery, Tagore Dental College and Hospital, Rathinamangalam,

Tamil Nadu, India.
Received: June 28, 2021

Published: August 05, 2021

(C) All rights are reserved by Jimson Samson., et al.

\begin{abstract}
Fracture of mandibular condyle represent $25 \%$ to $30 \%$ of all mandibular fractures and are thus among the most common facial fractures. There are variable pattern of fracture that may occur anywhere along the line from the sigmoid notch to the mandibular angle. It is often accompanied by fractures in other sites of the mandible. They differ from mandibular body fractures because they are more difficult to diagnose clinically and radiologically. Because of the importance of the condyle as the growth centre of the mandible and for lower accessibility, an accurate diagnosis and treatment of condyle fractures are very important. This review literature discussed about the various classifications of mandibular condylar fracture, the management options such as conservative management, open reduction internal fixation and the various open reduction managements.
\end{abstract}

Keywords: Fracture; Mandibular Condyle; Facial Fractures; Mandibular Angle

\section{Introduction}

Fracture of mandibular condyle represent $25 \%$ to $30 \%$ of all mandibular fractures and are thus among the most common facial fractures. There are variable pattern of fracture that may occur anywhere along the line from the sigmoid notch to the mandibular angle. It is often accompanied by fractures in other sites of the mandible. They differ from mandibular body fractures because they are more difficult to diagnose clinically and radiologically. Condyle boing a growth centre of mandible and the lesser accessibility makes it important to diagnose and treat condylar fractures accurately [1].
As condylar fractures are frequent and because condylar an important part of the TM Joint, various therapeutic methods have been described. The modalities of treatment can be divided into conservative (closed reduction) and surgical treatments (open reduction).

It has been reported that therapeutic methods undergone to treat these fractures produce satisfactory and functional outcomes $[2,3]$. Despite these good results, the choice between a conservative treatment or a surgical approach, open reduction and internal fixation (ORIF) remains a controversial topic [4]. 
Classification of mandibular condylar fracture

Wassmund 1927

Distinguishes the fractures of condylar head and the condylar neck. The condylar head fractures were classified as either comminated fractures or "chip fractures" not affecting the continuity.

The condylar neck fractures were further isolated to:

- Vertical neck fractures secondary to shearing.

- Transverse neck fractures secondary to bending.

- Oblique neck fractures result from combination of shearing/bending.

\section{Wassmund 1934}

Described dislocated fractures into three categories:

- Type I: 10 to 40 degree angulation of the condylar head with bony contact between the fragments.

- Type II: 50 to 90 degree angulation of the condylar head with less bony contact between the fragments.

- $\quad$ Type III: Severe and medially displaced with no contact between the bone fragments.

\section{MacLennan 1952 [5]}

Divided condylar fractures into sections consistent with anatomic location, the position of the condylar head within the articulating fossa, and also the association of between the larger/smaller fracture segments:

- Low condylar neck fracture line starts at the sigmoid notch and extends obliquely to the posterior border of the ramus.

- High condylar neck fracture starts above the sigmoid notch with involvement of the condylar neck.

- Subcondylar fractures is the posterior oblique fractures of the mandibular ramus.

- Avulsion of the condylar process seen in complete luxation fracture.

MacLennan further described the differences among simple "bending" of the condylar process, displacement fractures, and dislocation fractures:

- Class I: No deviation (bending).

- $\quad$ Class II: Deviation (bending) at the fracture level.
- Class III: Displacement (mandibular condylar head remains within fossa).

- Class IV: Dislocation (mandibular condylar head outside of fossa).

Rowe and Killey 1955 [6]

He simplified classification system based on the anatomy of the TMJ capsule and the surrounding structures of the TMJ:

- Intracapsular fractures

- Extracapsular fractures

- $\quad$ Fractures related to TMJ capsule, TMJ ligaments, articulating disk, and bony structures surrounding the TMJ.

Dingman and Natvig 1964 [6-8]

He proposed a classification system to include the insertion of the lateral pterygoid muscle at the condylar neck:

- High condylar neck fracture: fracture line is at or above the extend of the lateral pterygoid attachment on the fovea of the condylar apparatus.

- Intermediate condylar neck fracture: fracture line is below the extend of insertion of the lateral pterygoid.

- Low condylar neck fracture: fracture starts at or below the sigmoid notch and extends mandibular ramus posterior border.

Spiessl and Schroll 1972 [9]

He published a comprehensive manuscript. Commonly accepted in the European literature. They differentiated between fractures of the condylar base and neck, the degree of angulation associated with deviation, displacement, or dislocation

- $\quad$ Type I: Condylar neck fracture without deviation and/or displacement.

- Type II: Low condylar neck fracture with deviation and/or displacement.

- Type III: High condylar neck fracture with deviation and/ or displacement:

- IIIa: Ventral.

- IIIb: Medial.

- $\quad$ IIIc: Lateral. 
- IIId: Dorsal.

- $\quad$ Type IV: Low mandibular condylar neck fracture with dislocation.

- $\quad$ Type V: High mandibular condylar neck fracture with dislocation.

- $\quad$ Type VI: Intracapsular fracture of the mandibular condylar head.

\section{Lindahl $1977[10]$}

Published the most comprehensive classification of mandibular condylar head fractures to date within the literature. This classification system is highly descriptive. It is also complicated because it describes the location of the fracture, deviation, and/or displacement and position of the condylar head within the articulating fossa:

1. Fracture level:
a. Condylar head.
b. Condylar neck.
c. Subcondylar/condylar base.

2. Deviation and displacement:
a. Bending/deviation with medial overlapping segments.
b. Bending/deviation with medial overlapping segments.
c. Bending/displacement without overlapping.
d. Nondisplaced fracture without deviation

3. Relation between condylar head and fossa:
a. No dislocation.
b. Slight dislocation.
c. Moderate dislocation.
d. Severe and/or complete dislocation.

4. Condylar head fracture
a. Horizontal.
b. Vertical.
c. Compression fracture.

Lindahl defines the subcondylar fracture line as starting at the sigmoid notch and extending to the posterior border of the mandible. A condylar neck fracture is located below the level of the condylar head. Lindahl's classification system remains highly accurate, but difficult to recall because of the multiple subsections involved in a complete description of the fracture site.

\section{Modifications of Spiessl and Schroll}

Modifications to the descriptions of Spiessl and Schroll were conducted by various authors adding the component of condylar head integrity (diacapitular fracture) for type V and VI fractures. These included Rasse in 1993, Neff and coworkers in 1999, Hlawitschka and Eckelt in 2002, and Loukota and coworkers in 2010. The changes evolved into the following clarifications of the Spiessl and Schroll system:

- Type A: Continuous bony contact within the articular fossa, with a component of the condylar head remaining and the fracture supported without loss of ramus height.

- $\quad$ Type B: Loss of support within the articulating fossa and subsequent loss of mandibular ramus height.

- Type C: The uppermost portion of the fracture is below the level of the lateral ligament, resulting in a loss of ramus height.

\section{Ellis and coworkers 1999 [11]}

Described a more simplified classification system, which dealt with the location of the fracture and the degree of dislocation and/ or displacement:

- Condylar head fracture: Intracapsular fracture.

- Condylar neck fracture: Fracture below the condylar head, but on or above the lowest point of the sigmoid notch. 
- $\quad$ Condylar base fracture: Fracture in which the fracture line is located below the lowest point of the sigmoid notch.

- $\quad$ No detectable dislocation and correct positioning of the condylar head.

- $\quad$ Slight dislocation: Most of the condylar head remains within the articulating fossa and the degree of angulation/bending of the condylar process is less than 20 .

Loukota and coworkers $2005[11,12]$

Proposed a classification system for fractures of the condylar process of the mandible, which was subsequently adopted by the Strausbourg Osteosynthesis Research Group. This protocol described "Line A," which is a perpendicular line that extends through the lowest extension of the sigmoid notch to the mandibular ramus. The purpose of the line is to identify a component of the structural anatomy of the mandible that is easily reproducible even in cases of significant condylar trauma. Additionally, clarification of the condylar head fracture (diacapitular fracture) was noted, and presented a definition for the term "minimal displacement":

- Diacapitular fracture: The fracture line starts in the articular surface and may extend outside the TMJ capsule.

- Condylar neck: The fracture line starts somewhere above Line A and runs above Line A for more than half of its length.

- Condylar base: The fracture line extends behind the mandibular foramen and runs below Line A for more than half of its length.

- Minimal displacement: Displacement of less than 10 or overlap of the bone edges by less than $2 \mathrm{~mm}$, or both.

\section{A0 foundation expanded on Ellis classification 2010 [13]}

The AO Foundation expanded on Ellis' classification with the determination of "high-neck" and "low-neck" fractures within the online AO Surgery Reference in 2010, providing greater detail to the location of "high and low" as theorized by Loukota:

- $\quad$ The first line parallels the posterior border of the mandible.

- The sigmoid notch line runs perpendicular to the first line at the deepest portion of the sigmoid notch.
- There is a line below the lateral pole of the condylar head that is also perpendicular to the first line:

- A line is drawn half way between the lateral pole line and the sigmoid notch line.

- A "high-neck" fracture is above this line, whereas a "low-neck" fracture is below.

Neff and coworkers $2014[13,14]$

Published the Comprehensive AOCMF Classification System: Condylar Process Fractures. This system highlights numerous avenues of fracture location, identification, displacement, comminution, and dislocation. There is an attempt at clarity in identifying the location of the condylar fracture:

- Condylar head: The condylar head reference line runs perpendicular to the posterior ramus below the lateral pole of the condylar head.

- Condylar neck: The sigmoid notch line running through the deepest point of the sigmoid notch perpendicular to the ramus line extending superiorly to the condylar head.

- $\quad$ Base of the condylar process: The sigmoid notch line running through the deepest point of the sigmoid notch perpendicular to the ramus line extending inferiorly.

The operative surgeon should feel comfortable using the classification system that best delineates the location and description of the condylar fracture in a manner that affords the clearest understanding of the injury sustained by the patient. Once the determination has been made regarding the location and anatomic components of the injury, the surgeon can then appropriately discuss the inherent risks/benefits of open versus closed operative management with the patient and decide on a course of action.

\section{Closed reduction}

One of the main advantages of conservative treatment is the elimination of the risks involved in any surgical intervention. Nevertheless, the results are compromised mainly due to incorrect positioning of the fractured segments sometimes accompanied by complications such as chronic residual pain, articular and/or occlusal imbalance, ankylosis caused by prolonged immobilization or facial asymmetry resulted from shortened mandibular ramus $[15,16]$. 
In many cases, closed treatment is still an excellent option though many surgeons have moved away from traditional rigid fixation of 4 - 6 weeks. As the mechanism of repair of closed treatment is through neuromuscular adaptation rather than a return to correct anatomic position, rigid maxillomandibular fixation is typically used for no more than 7 - 10 days, with many surgeons placing guiding elastics at the time of closed treatment [17]. The elastics enable the patient to guide their bite back into natural occlusion while simultaneously initiating early physiotherapy and functional recovery while decreasing the risk of temporomandibular joint (TMJ) ankylosis. Closed management is best used to treat those patients with minimally displaced fractures who have difficulty coming into occlusion on their own.

\section{Open reduction with internal fixation}

On the other hand, ORIF presents the risks of surgical procedure, such as haemorrhage, infection, neurological or aesthetic risks arising from residual scarring. ORIF treatment requires professionals with higher levels of skill and training [18,19]. However, ORIF also offers considerable advantages: it leads to a better reduction and repositioning of the fractured fragments with an immediate mobilization and early recovery which circumvents many of the residual complications of conservative management [10].

Recent studies have suggested that open reduction with internal fixation (ORIF) leads to improved functional outcomes. The meta-analyses performed by Al-Moraissi and Ellis found that ORIF led to more mandibular mobility, a reduction in postoperative pain, and improved occlusion for patients undergoing open treatment [17]. The study by Chrcanovic also found that functional outcomes were superior for patients treated with ORIF when compared with closed management. Their study did show a statistically lower infection rate when closed treatment was utilized [20]. In the 2014 meta-analysis by Yao and Zhou, which included 859 patients, the authors noted that maximum mouth opening was significantly wider in those patients that underwent ORIF. At 1-year follow-up, the ORIF group was also noted to have lower rates of malocclusion. TMJ pain and facial asymmetry were similar in both cohorts $[20,21]$. Based on recent literature analysis, ORIF provides clear advantages in postoperative malocclusion and function. However, no advantage is seen with TMJ arthralgia.
The inevitable improvement in technology has advanced patient safety and surgical efficacy, thereby expanding the absolute indications for ORIF originally proposed by Zide and Kent [22]: displacement of the condyle into the middle cranial fossa, difficulty in obtaining adequate occlusion by closed reduction alone, and invasion of the condylar neck by a foreign body. Although these continue to be reasons to open the fracture site, both Ellis and Kell maintain the need for open treatment also in edentulous patients or those missing significant posterior dentition. The loss of vertical mandibular height that occurs in these cases cannot be adequately treated by closed reduction. This loss of height causes altered jaw mechanics with either deviation toward the fractured side or, in the case of bilateral fractures, open bite deformity [23].

Another indication for ORIF is the presence of midfacial fractures with associated condylar fractures. In the case of panfacial fractures, reestablishment of mandibular height creates a stable base from which to reset occlusion and rebuild the mid- face in a bottom to top manner.

The treatment of bilateral subcondylar fractures is more controversial and does not, in itself, mandate operative repair. Ellis found that $10 \%$ of patients in this cohort do not respond well to closed treatment. Unfortunately, it is unclear which patients will be recalcitrant to treatment. Many authors advocate the same approach to decision-making used in unilateral fracture management to treat these types of injuries.

\section{Approaches}

A search of the literature will encounter a multitude of surgical approaches to access the condylar region with each author expounding why their approach is optimal. As is the case when there are numerous solutions to a problem, there is more than one correct answer with each having its benefits and drawbacks. It is easiest to categorize approaches based on accessibility of mandibular regions along with its relationship to the facial nerve.

\section{Preauricular approach - Above the facial nerve}

The incision lies above the main trunk of the facial nerve and the condylar head and upper neck are accessible with this approach $[23,24]$. Fractures caudal to these areas can be accessed with downward skin retraction though at the risk of traction injury 
to the nerve. Mohan., et al. found, when compared with the retromandibular approach, the preauricular approach had more postoperative facial nerve weakness which they attributed to retraction injury to access lower neck fractures [25]. Cosmetically, the scar is well hidden within the preauricular crease.

\section{Submandibular approach - Below the nerve}

This approach provides excellent access to fractures of the ramus and low subcondylar areas. The skin incision is placed $2 \mathrm{~cm}$ below the angle of the mandible and when placed in a natural skin crease, gives a fairly reasonable cosmetic result. The main complication is either paralysis or paresis of the marginal mandibular branch of the facial nerve either from direct injury or from retraction forces [24].

Oral approach/endoscopic - Assisted reduction and internal fixation

Endoscopic-assisted reduction and internal fixation (ERIF) minimizes the major concerns of external approaches, including facial nerve injury, salivary fistula, and external scarring while providing the benefits of anatomic reduction [26]. In a prospective randomized multicenter trial, Schmelzeisen., et al. [27] compared ERIF with external approaches in 74 patients with displaced condylar neck fractures and found comparable functional results. Nogami., et al. $[27,28]$ found similar results when comparing ERIF to the retromandibular approach. There was a general tendency toward lower facial nerve palsy, however, this was not statistically significant. Patients who cannot tolerate maxillomandibular fixation or who have a mild functional discrepancy that can be restored with ERIF.

The disadvantages of ERIF include the need for dedicated instrumentation, steep learning curve, and well trained assistants to aid with the endoscope and retraction [27-29]. Comminuted fractures or those fractures where the proximal portion of the mandible is medially displaced may be difficult to reduce through a solely transoral endoscopic approach. Kang., et al. [30] reported on a series of 26 patients undergoing ERIF of mandibular condyle fractures. Eighteen patients had temporary complications, defined as those occurring within 3 months from surgery. These included: wound infection, TMJ disturbance, occlusal problems, and facial paraesthesia or palsy. Subsequently, six of these patients had complications beyond 3 months, including open bite, trismus, TMJ clicking, and TMJ pain.

\section{Angled drill}

One of the drawbacks of the endoscopic approach is that while fracture reduction and placement of the plate can be done transorally, a transcutaneous trocar is still required to drill holes and to place screws. Searching for an alternative that avoids external scars and risk to the facial nerve, some surgeons have moved to using an angled drill and screwdriver, obviating the need for transcutaneous instrumentation. Nam., et al. [30,31] treated 11 patients through an open approach with a small-angled drill. They found that the surgery time was similar to other approaches as their outcomes but without the risk to the facial nerve. Vajgel's group examined 25 subcondylar fractures and seven condylar neck fractures with a transoral approach using an angled drill [30-32]. At 2-year followup they found no joint noise, weakness of the facial nerve, joint pain, or muscle pain. The study by Schon., et al. in 2005 employed both endoscopic assistance as well as the use of an angled drill. As instrumentation improves both for scopes and drills, this may become the treatment approach for most subcondylar fractures [33].

\section{Pediatric fractures}

The condyle is the most common site of fracture in pediatric maxillofacial trauma [34]. Compared with their adult counterparts, pediatric condylar fractures are more commonly managed conservatively, and this has not changed significantly in recent years [5]. Ghasemzadeh., et al. [35] published their experience with 64 pediatric patients with 92 condylar fractures, all managed without open treatment. Although they had a low overall rate of complication, worse outcomes were identified in patients with comorbid mandibular arch fractures. These patients often required ORIF of their associated arch fracture, possibly indicating a more severe underlying injury. Traditionally, the use of conventional arch bars in pediatric patients during primary and mixed dentition was avoided, however recent studies show that this can be done safely without periodontal defects, tooth avulsions, or disruption to permanent dentition. In contrast, intermaxillary fixation screws should not be placed in the presence of uninterrupted teeth [36].

\section{Retromandibular approach}

Management of condylar fractures has been subjected to a lot of disagreement and debate. There is no general agreement for definitive treatment. In the past, condylar fractures were treated mostly using the closed reduction. The reason for this could be the surgical infection in the pre-antibiotic era and surgical approach to 
the anatomical location of these fractures will risk the facial nerve branches causing the facial nerve morbidity. Closed reduction and immobilization have complications such as occlusal dearrangement, pain, the prolonged period of maxillomandibular fixation affect the quality of life for patients $[37,38]$. Recent publications provide evidences that surgically treated condylar fractures have the better results regarding occlusion, masticatory function, mouth opening and bone morphology [39]. The decision to proceed with ORIF generates two other interrelated controversial issues namely, the type of fixation device that should be employed and the choice of surgical approach $[40,41]$. The level of condylar fracture anatomically, fixation method used, presence of other fractures, availability of surgical expertise and concerns regarding cosmetic all influence the selection of surgical approach. Open reduction and internal fixation of mandibular condylar fracture comes with various surgical approach. They are such as submandibular approach, retromandibular approach, preauricular approach etc. Each of these approaches has its own advantages and disadvantages $[41,42]$. Accessing the fracture line is sometimes unsatisfactory in the most popular approaches, such as the Risdon, especially in cases of high fracture because the skin incision is made far from the fracture site and so soft tissue retraction is not easy. Therefore, the screwdriver is used in an oblique direction to the bone surface. Under these conditions, getting stabilization is very difficult, and the construct may be deficient. This strong soft tissue retraction increases the risk of facial nerve damage that ranges from 30 - 48\% $[42,43]$. In another study the prevalence of facial nerve injury after surgical fracture treatment of the mandibular condyle ranges from $12 \%$ to $48 \%[44,45]$. Nerve damage may be resulted from dissection and retraction of the tissue, fracture fragments manipulation or hardware application [46].

Amongst the several approaches, the retromandibular transparotid approach is the most widely used one. The retromandibular approach was first described in 1967 Hinds and Girotti [47] and modified in 1978 Koberg and Momms [48]. Advantages reported in this approach include less facial nerve morbidity which can be identified and retracted under direct vision, minimal working distance from the incision to the fracture site, good exposure, aesthetically pleasing results from a less conspicuous scar and ease of fracture reduction/fixation $[22,49]$. Types of retromandibular approach are transparotid and anteroparotid Transmasseteric based on their dissection to the condyle and ramus. Originally, transpa- rotid approach was described for retromandibular incisions [16]. The approach requires dissection of the parotid capsule and parenchyma of the parotid to reach the fracture site. In retromandibular transparotid approach, incision is placed on parotid fascia and then blunt dissection is carried out in the surface of the parotid gland parallel along the expected course of facial nerve branches to reach the pterygomasseteric sling [44,50]. The TMAP approach described by Wilson., et al. [9] in response to concerns about injury to the facial nerve and parotid-related complications resulting from the transparotid route. It is easy technique to learn, provides adequate surgical exposure for open reduction internal fixation, and has minimal complications rates. Wilson., et al. described a transmasseteric anteroparotid approach for management of sub condylar fracture through preauricular incision extended in curvilinear fashion in cervico mastoid crease. The dissection is carried out superficial to the parotid capsule till the anterior portion of the gland, masseter muscle is identified and vertical incision is given in masseter muscle and dissection is carried out along the fibers of masseter muscle to reach the periosteum. The length of incision was longer in their approach and it leaves the scar in the preauricular region [51].

\section{Conclusion}

Since the first description of TMAP, the approach has been modified by a number of authors [52,53]. The variations are in the length and size of the superior extension of the incision, with some authors restricting it to the lobule while others extend it as far as to the tragus $[54,55]$.

\section{Bibliography}

1. Kim B-K., et al. "Usefulness of the retromandibular transparotid approach for condylar neck and condylar base fractures". The Journal of Craniofacial Surgery 23.3 (2012): 712-715.

2. Booth PW., et al. "Maxillofacial Surgery". Churchill Livingstone (2007): 1760.

3. Marker P., et al. "Fractures of the mandibular condyle. Part 2: results of treatment of 348 patients". British Journal of Oral and Maxillofacial Surgery 38.5 (2000): 422-426.

4. Gerbino G., et al. "Long-Term Clinical and Radiological Outcomes for the Surgical Treatment of Mandibular Condylar Fractures". Journal of Oral and Maxillofacial Surgery 67 (2009): 1009-1014. 
5. MacL Ennan Wd. "Consideration of 180 cases of typical fractures of the mandibular condylar process". British Journal of Plastic Surgery 5.2 (1952): 122-128.

6. NL Rowe., et al. "Fractures of the facial skeleton". Consultants in Oral Surgery, Plastic and Maxillofacial Surgery Unit, Rooksdown House, Basingstoke. With a Foreword by Sir Reginald Watson-Jones. 9 3/4 × 6 3/4 in. Pp. 923 xxxvi, with 1231 illustrations, 14 in colour. 1955. Edinburgh: E. and S. Livingstone Ltd. £6". British Journal of Surgery 43 (2005): 446-446.

7. Kilner TP., et al. Consultants in Oral Surgery, Plastic and Maxillo-facial Surgery Unit, Rooksdown House, Basingstoke. With a foreword by Sir Reginald Watson-Jones, and contributions from eight other authors. 10x7 $\hat{A} 1 / 2$ in. Pp. xxxvi 923, with 1,231 figures, 14 in full colour. Index. 1955. Edinburgh and London: E. \& S. Livingstone Ltd. Price Â£6". The Journal of Bone and Joint Surgery British Volume 39-b (1957): 806-806.

8. By Reed O Dingman., et al. "Surgery of facial fractures. $10 \times 7 \frac{1}{2}$ in. Pp. 380. Heavily illustrated, 1964. London: W. B. Saunders Ltd. $€ 7$ 17s". British Journal of Surgery 51 (2005): 560-560.

9. Spezielle Frakturen und Luxationslehre: Gesichts schadel. Edited by H. Nigst, Basel. $10 \times 7$ in. Pp. 213 xii. Illustrated. 1972 . Stuttgart: Georg Thieme Verlag. DM.164". British Journal of Surgery 60 (2005): 335-335.

10. Lindahl L and Hollender L. "Condylar fractures of the mandible”. International Journal of Oral Surgery 6 (1977): 153-165.

11. Ellis E., et al. "Further displacement of condylar process fractures after closed treatment". Journal of Oral and Maxillofacial Surgery 57 (1999): 1307-1316.

12. Loukota RA., et al. "Subclassification of fractures of the condylar process of the mandible". British Journal of Oral and Maxillofacial Surgery 43 (2005): 72-73.

13. Website [Internet]. [cited 2021 May 27]. Available from: https://www2.aofoundation.org/wps/portal/!ut/p/ a1/04_Sj9CPykssy0xPLMnMz0vMAfGjzOKN_A0M3D2DDbz9_UMMDRyDXQ3 dw9w MDAwCTYEKIvEocDQnTr8BDuBoQEi_l35Uek5 -EtCp4c76US6huYZ-6SBDHfOSjC3S9aOKUtNSi1 KL9DLyi0v0I8rLy430EvPT8kvzUsBe1MsvStcvyA2 NqPIsdgQA63nsuA!!/dl5/d5/ L 2 d J Q S Ev UUt 3 Q S 80 S m lF L 1 o 2 X z J P M D B H S V M wS09PVDEwQVNFMUdWRjAwMFE1/?boneZCMF\&segmentZ Mandible\&showPageZdiagnosis.
14. Neff A., et al. "The Comprehensive AOCMF Classification System: Condylar Process Fractures - Level 3 Tutorial”. Craniomaxillofacial Trauma and Reconstruction: SAGE Journals 7.1 (2014): S044-58.

15. Choi J., et al. "A follow-up study of condyle fracture in children". International Journal of Oral and Maxillofacial Surgery 34.8 (2005): 851-858.

16. Thorén H., et al. "Condylar process fractures in children: a follow-up study of fractures with total dislocation of the condyle from the glenoid fossa". Journal of Oral and Maxillofacial Surgery 59.7 (2001): 773-774.

17. Al-Moraissi EA and Ellis E. "Surgical Treatment of Adult Mandibular Condylar Fractures Provides Better Outcomes Than Closed Treatment: A Systematic Review and Meta-Analysis". Journal of Oral and Maxillofacial Surgery 73 (2015): 482-493.

18. Eckelt U., et al. "Open versus closed treatment of fractures of the mandibular condylar process-a prospective randomized multi-centre study". Journal of Craniofacial Surgery 34.5 (2006): 306-314.

19. Jensen T., et al. "Open reduction and rigid internal fixation of mandibular condylar fractures by an intraoral approach: a long-term follow-up study of 15 patients". Journal of Oral and Maxillofacial Surgery 64.12 (2006): 1771-1779.

20. Chrcanovic BR. "Surgical versus non-surgical treatment of mandibular condylar fractures: a meta-analysis". International Journal of Oral and Maxillofacial Surgery 44 (2015): 158-179.

21. Yao S., et al. "Contrast Analysis of Open Reduction and Internal Fixation and Non-Surgical Treatment of Condylar Fracture". Journal of Craniofacial Surgery 25 (2014): 2077-2080.

22. Zide MF and Kent JN. "Indications for open reduction of mandibular condyle fractures". Journal of Oral and Maxillofacial Surgery 41 (1983): 89-98.

23. Ellis E., et al. "Subcondylar Fractures". Facial Plastic Surgery Clinics of North America 20 (2012): 365-382.

24. Sawhney R., et al. "Condylar fractures". Otolaryngologic Clinics of North America 46.5 (2013): 779-790.

25. Mohan AP., et al. "Comparison of preauricular approach versus retromandibular approach in management of condylar fractures". Journal of Oral and Maxillofacial Surgery 11.4 (2012): 435-441. 
26. Hackenberg B., et al. "Management of subcondylar mandible fractures in the adult patient". The Journal of Craniofacial Surgery 25.1 (2014): 166-171.

27. Schmelzeisen R., et al. "Patient Benefit From Endoscopically Assisted Fixation of Condylar Neck Fractures-A Randomized Controlled Trial". Journal of Oral and Maxillofacial Surgery 67 (2009): 147-158.

28. Nogami S., et al. "Clinical comparison between the retromandibular approach for reduction and fixation and endoscopeassisted open reduction and internal fixation for mandibular condyle fractures". The Journal of Craniofacial Surgery 23.6 (2012): 1815-1818.

29. Sawhney R., et al. "Endoscopic Treatment of Subcondylar Fractures". Current Otorhinolaryngology Reports 3 (2015): 226232.

30. Kang S-H., et al. "Complications in endoscopic-assisted open reduction and internal fixation of mandibular condyle fractures". Oral Surgery, Oral Medicine, Oral Pathology, Oral Radiology, and Endodontology 113.2 (2012): 201-206.

31. Nam SM., et al. "Transoral Open Reduction for Subcondylar Fractures of the Mandible Using an Angulated Screwdriver System". Annals of Plastic Surgery 75.3 (2015): 295-301.

32. Vajgel A., et al. "Treatment of condylar fractures with an intraoral approach using an angulated screwdriver: results of a multicentre study". Journal of Cranio-Maxillofacial Surgery 43.1 (2015): 34-42.

33. Keefe MA. "Five-Year Experience with the Transoral Endoscopically Assisted Treatment of Displaced Condylar Mandible Fractures". Yearbook of Otolaryngology-Head and Neck Surgery (2007): 165-166.

34. Boffano P., et al. "European Maxillofacial Trauma (EURMAT) in children: a multicenter and prospective study". Oral Surgery, Oral Medicine, Oral Pathology, Oral Radiology, and Endodontology 119.5 (2015): 499-504.

35. Ghasemzadeh A., et al. "Treatment of Pediatric Condylar Fractures: A 20-Year Experience". Plastic and Reconstructive Surgery 136.6 (2015): 1279-1288.

36. Naran S., et al. "The safe and efficacious use of arch bars in patients during primary and mixed dentition: a challenge to conventional teaching". Plastic and Reconstructive Surgery 133.2 (2014): 364-366.
37. Singh V., et al. "Outcomes of open versus closed treatment of mandibular subcondylar fractures: a prospective randomized study". Journal of Oral and Maxillofacial Surgery 68.6 (2010): 1304-1309.

38. Zachariades N., et al. "Fractures of the mandibular condyle: a review of 466 cases. Literature review, reflections on treatment and proposals". Journal of Cranio-Maxillofacial Surgery 34.7 (2006): 421-432.

39. Biglioli F and Colletti G. "Mini-retromandibular approach to condylar fractures". Journal of Cranio-Maxillofacial Surgery 36.7 (2008): 378-383.

40. Haug RH and Brandt MT. "Closed reduction, open reduction, and endoscopic assistance: current thoughts on the management of mandibular condyle fractures". Plastic and Reconstructive Surgery 120.7-2 (2007): 90S-102S.

41. Emam HA., et al. "Matching Surgical Approach to Condylar Fracture Type". Atlas of the Oral and Maxillofacial Surgery Clinics of North America 25.1 (2017): 55-61.

42. Kempers KG., et al. "Surgical approaches to mandibular condylar fractures: a review". The Journal of Cranio-Maxillofacial Trauma 5.4 (1999): 25-30.

43. Choi BH and Yoo JH. "Open reduction of condylar neck fractures with exposure of the facial nerve". Oral Surgery, Oral Medicine, Oral Pathology, Oral Radiology, and Endodontology 88.3 (1999): 292-296.

44. Downie JJ., et al. "Prospective study of morbidity associated with open reduction and internal fixation of the fractured condyle by the transparotid approach". British Journal of Oral and Maxillofacial Surgery 47.5 (2009): 370-373.

45. Yang L and Patil PM. "The retromandibular transparotid approach to mandibular subcondylar fractures". International Journal of Oral and Maxillofacial Surgery 41 (2012): 494-499.

46. Yamamoto MK., et al. "Evaluation of surgical retreatment of mandibular fractures". Journal of Cranio-Maxillofacial Surgery 41 (2013): 42-46.

47. Hinds EC and Girotti WJ. "Vertical subcondylar osteotomy: A reappraisal”. Oral Surgery, Oral Medicine, Oral Pathology 24 (1967): 164-170.

48. Koberg WR and Momma WG. "Treatment of fractures of the articular process by functional stable osteosynthesis using miniaturized dynamic compression plates". International Journal of Oral and Maxillofacial Surgery 7.4 (1978): 256-262. 
49. Manisali M., et al. "Retromandibular approach to the mandibular condyle: a clinical and cadaveric study". International Journal of Oral and Maxillofacial Surgery 32.3 (2003): 253-256.

50. Devlin MF., et al. "Open reduction and internal fixation of fractured mandibular condyles bya retromandibular approach: surgical morbidity and informed consent". British Journal of Oral and Maxillofacial Surgery 40 (2002): 23-25.

51. Wilson AW., et al. "Transmasseteric antero-parotid approach for open reduction and internal fixation of condylar fractures". British Journal of Oral and Maxillofacial Surgery 43.1 (2005): 57-60.

52. Salgarelli AC., et al. "How to improve retromandibular transmasseteric anteroparotid approach for mandibular condylar fractures: our clinical experience". International Journal of Oral and Maxillofacial Surgery 42.4 (2013): 464-469.

53. Tang W., et al. "Application of Modified Retromandibular Approach Indirectly From the Anterior Edge of the Parotid Gland in the Surgical Treatment of Condylar Fracture". Journal of Oral and Maxillofacial Surgery 67 (2009): 552-558.

54. Ebenezer V and Ramalingam B. "Comparison of Approaches for the Rigid Fixation of Sub-Condylar Fractures". Journal of Maxillofacial and Oral Surgery 10 (2011): 38-44.

55. Narayanan V., et al. "Transmasseteric anterior parotid approach for condylar fractures: experience of 129 cases". British Journal of Oral and Maxillofacial Surgery 50.5 (2012): 420-424.

\section{Volume 5 Issue 9 September2021}

(C) All rights are reserved by Jimson Samson., et al. 